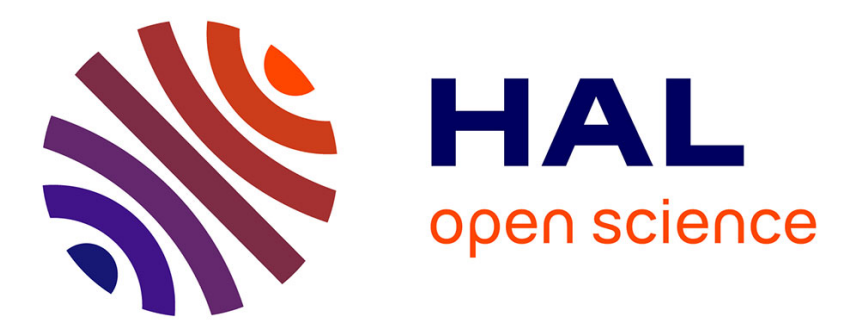

\title{
On the controllability of the improved Boussinesq equation *
}

Eduardo Cerpa, Emmanuelle Crépeau

\section{To cite this version:}

Eduardo Cerpa, Emmanuelle Crépeau. On the controllability of the improved Boussinesq equation *. 2017. hal-01661174

\section{HAL Id: hal-01661174 https://hal.science/hal-01661174}

Preprint submitted on 11 Dec 2017

HAL is a multi-disciplinary open access archive for the deposit and dissemination of scientific research documents, whether they are published or not. The documents may come from teaching and research institutions in France or abroad, or from public or private research centers.
L'archive ouverte pluridisciplinaire HAL, est destinée au dépôt et à la diffusion de documents scientifiques de niveau recherche, publiés ou non, émanant des établissements d'enseignement et de recherche français ou étrangers, des laboratoires publics ou privés. 


\title{
On the controllability of the improved Boussinesq equation*
}

\author{
Eduardo $\mathrm{Cerpa}^{\dagger}$ and Emmanuelle Crépeau ${ }^{\ddagger}$
}

\begin{abstract}
The improved Boussinesq equation is studied in this paper. Control properties for this equation posed on a bounded interval are first considered. When the control acts through the Dirichlet boundary condition the linearized system is proved to be approximately but not spectrally controllable. In a second part, the equation is posed on the one-dimensional torus and distributed moving controls are considered. Under some condition on the velocity to which the control moves, exact controllability results for both linear and nonlinear improved Boussinesq equations are obtained applying the moment method and a fixed point argument.
\end{abstract}

Key words. Boussinesq type equation, exact controllability, approximate controllability, spectral analysis, moving control, moment method, fixed point theorem

AMS subject classifications. 35Q35, 93B05, 93C10

\section{Introduction}

In [1], Boussinesq derived the so called "bad" Boussinesq equation, written

$$
y_{t t}-y_{x x}-y_{x x x x}=\left(y^{2}\right)_{x x} .
$$

This equation describes the flow of shallow water waves with small amplitudes in a flat bottom canal. It is called "bad" due to its poor existence and uniqueness properties. For instance, unlike the "good" Boussinesq equation, which reads as

$$
y_{t t}-y_{x x}+y_{x x x x}=\left(y^{2}\right)_{x x},
$$

there is no local well-posedness results for equation (1). However, in [12, Makhankov proved that the "bad" Boussinesq equation (1) can be approached by the following one, called improved Boussinesq equation,

$$
y_{t t}-y_{x x}-y_{x x t t}=\left(y^{2}\right)_{x x} .
$$

The well-posedness problem for the improved Boussinesq equation (3) with Dirichlet boundary conditions has been studied by Zhijian in [20].

Concerning the control of these equations, due to the lack of a well-posedness framework, there is no control results dealing with the "bad" Boussinesq equation. On the

\footnotetext{
* This work has been partially supported by Fondecyt 1140741, MathAmsud COSIP, and CONICYTBasal Project FB0008 AC3E.

${ }^{\dagger}$ Departamento de Matemática, Universidad Técnica Federico Santa María, Avda. España 1680, Valparaíso, Chile. E-mail: eduardo.cerpa@usm.cl.

${ }^{\ddagger}$ Laboratoire de Mathématiques, UVSQ, CNRS, Université Paris-Saclay, 78035 Versailles, France. E-mail: emmanuelle.crepeau@uvsq.fr.
} 
other hand, the boundary controllability for the "good" Boussinesq equation (2) posed on a bounded domain was addressed in [6]. In that paper, a local controllability result for the nonlinear equation is obtained with the help of the Hilbert Uniqueness Method for the controllability of the linearized equation and a fixed point theorem to obtain the local controllability of the nonlinear one.

In this paper, we are concerned with the controllability of the improved Boussinesq equation (3) posed either on a bounded or periodic domain. In the case of a bounded domain $[0,1]$ with boundary control, we prove that the linearized equation is not spectrally controllable, and consequently, not null controllable. Despite these negative results, we prove an approximate controllability result. Those results of non controllability are due to the existence of a finite limit point in the spectrum. Previous results of bad control properties due to the spectrum have already been noticed by Russell [18] for the beam equation with internal damping, by Leugering and Schmidt [10] for the plate equation. For our study we strongly use the articles written by Micu [14 for the linearized Benjamin-Bona-Mahony (BBM) equation and by Rosier and Rouchon [15] for the structurally damped wave equation.

In order to improve the control properties of our equation, we study the improved Boussinesq equation (3) posed on a periodic domain and we consider a moving distributed control. This kind of moving actuators has been considered previously in the literature since the work [11] by Lions. In that paper, the wave equation with moving point control was considered (see also [8]). For the same equation, we find the more recent papers [2, 3, 13, 5]. Concerning parabolic equations, we can cite the papers [7, 4] dealing with semi linear and linear heat equations. In [16, Rosier and Zhang proved that the BBM equation posed in the torus with a moving distributed control is locally exactly controllable for a control time large enough.

In the second part of this paper, we are able to prove the local exact controllability of the improved Boussinesq equation under a condition on the velocity the control moves. The controllability is proved with the moment method for the linearized equation, and then with a fixed point argument in order to deal with the nonlinearity, as our equation inherits some important properties of the BBM equation we use the ideas of the proofs in [16] and [13] to obtain our results.

The rest of this paper is organized as follows. In Section 2 we consider the linearized improved Boussinesq equation posed on a bounded domain with a boundary control. The approximate controllability and the lack of exact controllability are proved. The periodic case with a moving control is studied in Section 3, where exact control results are obtained for both linear and nonlinear improved Boussinesq equations.

\section{Boundary control on a bounded domain}

In this section we look at the boundary controllability of the linearized improved Boussinesq equation posed on the finite interval $[0,1]$. Namely, given a time $T>0$, an initial condition $\left(y^{0}, y^{1}\right)$ and a target $\left(y_{T}^{0}, y_{T}^{1}\right)$ on an appropriate space, we wonder if we can find a control function $h=h(t)$ such that the solution of the following linear problem

$$
\begin{cases}y_{t t}-y_{x x}-y_{x x t t}=0, & (x, t) \in(0,1) \times(0, T), \\ y(0, t)=0, y(1, t)=h(t), & t \in(0, T), \\ y(x, 0)=y^{0}(x), y_{t}(x, 0)=y^{1}(x), & x \in(0,1),\end{cases}
$$

satisfies $y(T)=y_{T}^{0}$ and $y_{t}(T)=y_{T}^{1}$. 


\subsection{Well-posedness}

We first look at the well-posedness of the homogeneous improved Boussinesq problem on a bounded domain

$$
\begin{cases}y_{t t}-y_{x x}-y_{x x t t}=0, & (x, t) \in(0,1) \times(0, T), \\ y(0, t)=y(1, t)=0, & t \in(0, T), \\ y(x, 0)=y^{0}(x), y_{t}(x, 0)=y^{1}(x), & x \in(0,1) .\end{cases}
$$

The well posedness of this problem has already been studied by Zhijian in [20]. He proved that the equation (5) with a nonlinear term $\left(y^{2}\right)_{x x}$ is well posed, locally in time, for $\left(y^{0}, y^{1}\right) \in\left(H^{2}(0,1) \cap H_{0}^{1}(0,1)\right)^{2}$ and the solution belongs to $C^{2}\left(\left[0, T_{0}\right), H^{2}(0,1) \cap\right.$ $\left.H_{0}^{1}(0,1)\right)$. We will establish this kind of results by using spectral methods for the linear equation (5).

We can rewrite the homogeneous system (5) as follows,

$$
y_{t t}+A y=0,
$$

where, for $D(A)=H^{2}(0,1) \cap H_{0}^{1}(0,1)$, we define the operator $A: D(A) \subset L^{2}(0,1) \longrightarrow$ $L^{2}(0,1)$ by means of

$$
A: w \longmapsto-\left(I-\partial_{x x}\right)^{-1} \partial_{x x} w .
$$

Proposition 1 There exists an orthonormal basis of $L^{2}(0,1)$ formed by eigenfunctions $\left\{f_{k}\right\}_{k \in \mathbb{N}^{*}}$ of the operator $A$. Moreover, this family is given by $f_{k}(x)=\sqrt{2} \sin (k \pi x)$ and the corresponding eigenvalues are $\lambda_{k}=\frac{k^{2} \pi^{2}}{k^{2} \pi^{2}+1}$, for any $k \in \mathbb{N}^{*}$.

Proof. Let $\lambda \in \mathbb{C}$ and $y \in H^{2}(0,1) \cap H_{0}^{1}(0,1)$ such that $A y=\lambda y$, then

$$
\left\{\begin{array}{l}
y_{x x}=-\lambda\left(y-y_{x x}\right) \\
y(0)=y(1)=0
\end{array}\right.
$$

From this, we see that

$$
\left\{\begin{array}{l}
y_{x x}=\frac{\lambda}{\lambda-1} y \\
y(0)=y(1)=0
\end{array}\right.
$$

and then, the eigenvalues are $\lambda_{k}=\frac{k^{2} \pi^{2}}{k^{2} \pi^{2}+1}$ and the corresponding eigenfunctions are $f_{k}(x)=\sqrt{2} \sin (k \pi x)$, for $k \in \mathbb{N}^{*}$.

Remark 2 We can easily remark that the eigenvalues $\lambda_{k} \in \mathbb{R}^{+}$are simple, and in addition $\lim _{k \rightarrow+\infty} \lambda_{k}=1$. Thus, the spectrum of $A$ admits a finite limit point.

By using the spectral decomposition of $A$ and the asymptotic behavior of $\lambda_{k}$, we can write the solutions of system (5) in the space $\mathcal{H}^{s}(0,1)$ defined in the following classical way for any $s \geq 0$ :

$$
\mathcal{H}^{s}(0,1)=\left\{\sum_{k \geq 1} a_{k} f_{k}(x) / \sum_{k \geq 1} k^{2 s}\left|a_{k}\right|^{2}<\infty\right\} .
$$

We can remark that: 
- for $s \leq 1 / 2, \mathcal{H}^{s}(0,1)=H^{s}(0,1)$,

- for $1 / 2<s \leq 3 / 2, \mathcal{H}^{s}(0,1)=H_{0}^{s}(0,1)$,

- and for $3 / 2<s \leq 2, \mathcal{H}^{s}(0,1)=H^{s}(0,1) \cap H_{0}^{1}(0,1)$,

where $H^{s}(0,1)$ and $H_{0}^{s}(0,1)$ are the usual Sobolev spaces. Thus, we explicitely obtain the following.

Proposition 3 Let $s \geq 0$. For every $\left(y^{0}, y^{1}\right) \in \mathcal{H}^{s}(0,1)^{2}$ the solution $y$ of equation (5) belongs to $C^{1}\left(\left[0,+\infty\left[, \mathcal{H}^{s}(0,1)\right)\right.\right.$ and can be written as

$$
y(x, t)=\sum_{k \geq 1}\left(a_{k} \cos \left(\sqrt{\lambda_{k}} t\right)+\frac{b_{k}}{\sqrt{\lambda_{k}}} \sin \left(\sqrt{\lambda_{k}} t\right)\right) f_{k}(x),
$$

where $y^{0}=\sum_{k \geq 1} a_{k} f_{k}$ and $y^{1}=\sum_{k \geq 1} b_{k} f_{k}$.

Remark 4 From the cosine and sine functions, we see that there is no gain of regularity for the linear improved Boussinesq equation. Moreover, from the asymptotic behavior of eigenvalues $\lambda_{k}$, we see the fact that the position $y$ and the velocity $y_{t}$ have the same regularity.

Remark 5 As we will see later, we need to have solutions y of the homogeneous problem such that the trace $y_{x}(1, t)$ exists as a function. From the previous proposition, we see that if $s>3 / 2$, then we obtain a solution such that the desired trace is a function. Another way to see that is through the following simple computation,

$$
\begin{aligned}
& \left|y_{x}(1, t)\right| \leq 2 \pi \sqrt{2} \sum_{n \geq 1}\left(n\left|a_{n}\right|+n\left|b_{n}\right|\right) \\
& \leq 2 \pi \sqrt{2}\left(\sum_{n \geq 1} n^{2-2 s}\right)^{1 / 2}\left(\sum_{n \geq 1} n^{2 s}\left|a_{n}\right|^{2}\right)^{1 / 2}+2 \pi \sqrt{2}\left(\sum_{n \geq 1} n^{2-2 s}\right)^{1 / 2}\left(\sum_{n \geq 1} n^{2 s}\left|b_{n}\right|^{2}\right)^{1 / 2},
\end{aligned}
$$

which gives the desired result when we are in regularity $\mathcal{H}^{s}(0,1)$ with $s>3 / 2$.

We are concerned now with the initial boundary value problem (4).

Proposition 6 Let $y^{0}, y^{1} \in L^{2}(0,1)$ and $h \in H^{2}(0, T)$. Then, the equation (4) has a unique mild solution $y$ in the space $C^{1}\left([0, T], L^{2}(0,1)\right)$.

Proof. If $y$ is the solution of (4), then $\varphi(x, t):=y(x, t)-x h(t)$ is the solution of

$$
\begin{cases}\varphi_{t t}-\varphi_{x x}-\varphi_{x x t t}=-x \ddot{h}, & (x, t) \in(0,1) \times(0, T), \\ \varphi(0, t)=\varphi(1, t)=0, & t \in(0, T), \\ \varphi(x, 0)=y^{0}-x h(0), \varphi_{t}(x, 0)=y^{1}-x \dot{h}(0), & x \in(0,1),\end{cases}
$$

where $\dot{h}$ and $\ddot{h}$ denote the first and second derivative in time of $h$, respectively.

By introducing $v=\varphi$ and $w=\varphi_{t}$, we can write this equation as a first order system as follows,

$$
\left\{\begin{aligned}
\frac{d}{d t}\left(\begin{array}{c}
v \\
w
\end{array}\right) & =\left(\begin{array}{cc}
0 & I \\
\left(I-\partial_{x x}\right)^{-1} \partial_{x x} & 0
\end{array}\right)\left(\begin{array}{c}
v \\
w
\end{array}\right)+\left(\begin{array}{c}
0 \\
-\ddot{h}\left(I-\partial_{x x}\right)^{-1} x
\end{array}\right), \\
\left(\begin{array}{c}
v(0) \\
w(0)
\end{array}\right) & =\left(\begin{array}{l}
y^{0}-x h(0) \\
y^{1}-x \dot{h}(0)
\end{array}\right) .
\end{aligned}\right.
$$


The forcing term belongs to $L^{2}\left(0, T ; \mathcal{H}^{s}(0,1)\right)$, for any $0 \leq s \leq 1 / 2$ (notice that the function $f(x)=x$ belongs to $\mathcal{H}^{s}(0,1)$ for any $\left.s \leq 1 / 2\right)$. Thus, by using a classical result, we obtain a well-posedness result for 111$)$ giving solutions $\varphi$ in $C^{1}\left([0, T], L^{2}(0,1)\right)$. Going back to $y$ we obtain the desired result (see [9, page 13] for a similar argument).

\subsection{Lack of exact controllability}

We want to study the exact controllability of

$$
\begin{cases}y_{t t}-y_{x x}-y_{x x t t}=0, & (x, t) \in(0,1) \times(0, T) \\ y(0, t)=0, y(1, t)=h(t), & t \in(0, T) \\ y(x, 0)=y^{0}(x), y_{t}(x, 0)=y^{1}(x), & x \in(0,1)\end{cases}
$$

where $h \in H^{2}(0, T)$ is the boundary control and $y^{0}, y^{1} \in L^{2}(0,1)$ are the initial data. Let $z$ be the solution of the adjoint problem of (12), which is given by

$$
\begin{cases}z_{t t}-z_{x x}-z_{x x t t}=0, & (x, t) \in(0,1) \times(0, T), \\ z(0, t)=z(1, t)=0, & t \in(0, T) \\ z(x, T)=z_{T}^{0}(x), z_{t}(x, T)=z_{T}^{1}(x), & x \in(0,1)\end{cases}
$$

for $z_{T}^{0}, z_{T}^{1} \in H^{2} \cap H_{0}^{1}(0,1)=\mathcal{H}^{2}(0,1)$. We decompose the initial data of $z$ in Fourier series,

$$
z_{T}^{0}=\sum_{n \geq 1} \tilde{a}_{n} f_{n}(x), z_{T}^{1}=-\sum_{n \geq 1} \tilde{b}_{n} f_{n}(x)
$$

with $\sum_{n \geq 1}\left(n^{4}\left|\tilde{a}_{n}\right|^{2}+n^{4}\left|\tilde{b}_{n}\right|^{2}\right)<\infty$, in order to write the solution of $(13)$ as

$$
z(x, t)=\sum_{n \geq 1}\left(\tilde{a}_{n} \cos \left(\sqrt{\lambda_{n}}(T-t)\right)+\frac{\tilde{b}_{n}}{\sqrt{\lambda_{n}}} \sin \left(\sqrt{\lambda_{n}}(T-t)\right)\right) f_{n}(x) .
$$

Equivalently, in its complex form, this solution is given by

$$
z(x, t)=\sum_{n \geq 1}\left(\tilde{c}_{n} e^{i \sqrt{\lambda_{n}}(T-t)}+\tilde{d}_{n} e^{-i \sqrt{\lambda_{n}}(T-t)}\right) f_{n}(x)
$$

where

$$
\tilde{c}_{n}=\frac{1}{2}\left(\tilde{a}_{n}-\frac{i \tilde{b}_{n}}{\sqrt{\lambda_{n}}}\right) \text { and } \tilde{d}_{n}=\frac{1}{2}\left(\tilde{a}_{n}+\frac{i \tilde{b}_{n}}{\sqrt{\lambda_{n}}}\right) .
$$

We are now in a position to prove the following non controllability result.

Theorem 7 The control system 12 is not spectrally controllable in $L^{2}(0,1)$.

Proof. We prove that no nontrivial finite combination of eigenfunctions $\left\{f_{n}\right\}_{n \geq 1}$ can be driven to zero in finite time. Let $\left\{a_{n}\right\}_{n \geq 1}$ and $\left\{b_{n}\right\}_{n \geq 1}$ be two sequences of real numbers such that there exists $N \in \mathbb{N}$ with $a_{n}=b_{n}=0$ for all $n>N$.

Suppose that the system $(12)$ is spectrally controllable. Then, there exists a boundary control $h \in H^{2}(0, T)$ such that the solution of $(12)$ with initial data

$$
y^{0}=\sum_{n \geq 1} a_{n} f_{n}, \quad y^{1}=\sum_{n \geq 1} b_{n} f_{n}
$$


satisfies $y(T)=y_{t}(T)=0$.

We multiply 12 by $z$ and integrate in space and time over $(0,1) \times(0, T)$. Thus, we obtain

$$
\int_{0}^{1}\left[y^{1}\left(z(x, 0)-z_{x x}(x, 0)\right)-y^{0}\left(z_{t}(x, 0)-z_{x x t}(x, 0)\right)\right] d x=\int_{0}^{T}(h(t)+\ddot{h}(t)) z_{x}(1, t) d t
$$

for any solution $z$ of (13). Using this equation with appropriate trajectories, first with $z(x, t)=e^{i \sqrt{\lambda}_{n}(T-t)} f_{n}(x)$ and next with $z(x, t)=e^{-i \sqrt{\lambda}_{n}(T-t)} f_{n}(x)$, we have that the control $h$ is the solution of the moment problem composed, for any $n \geq 1$, of the following equations

$$
\left\{\begin{array}{c}
\left(1+n^{2} \pi^{2}\right)\left(i \sqrt{\lambda_{n}} a_{n}+b_{n}\right)=\sqrt{2}(n \pi)(-1)^{n} \int_{0}^{T}(h(t)+\ddot{h}(t)) e^{-i \sqrt{\lambda_{n}} t} d t \\
\left(1+n^{2} \pi^{2}\right)\left(-i \sqrt{\lambda_{n}} a_{n}+b_{n}\right)=\sqrt{2}(n \pi)(-1)^{n} \int_{0}^{T}(h(t)+\ddot{h}(t)) e^{i \sqrt{\lambda_{n}} t} d t .
\end{array}\right.
$$

We proceed as in [14] and [15]. Let us define the complex function

$$
F(z):=\int_{0}^{T}(h(t)+\ddot{h}(t)) e^{i z t} d t
$$

Due to the Paley-Wiener theorem, $F$ is an entire function and it satisfies $F\left( \pm \sqrt{\lambda_{n}}\right)=0$, for all $n>N$. Because the asymptotic behavior of the eigenvalues $\left(\sqrt{\lambda_{n}} \rightarrow 1\right.$ as $\left.n \rightarrow \infty\right)$, we see that $F$ vanishes on a set with a finite accumulation point. Therefore, we conclude that $F \equiv 0$. From (15), we easily obtain that $a_{n}=b_{n}=0$ for each $n \geq 1$. That means that the trivial state is the only one which can be steered to zero.

Remark 8 System (12) being not spectrally controllable, we consequently know that (12) is neither exact nor null controllable. This is clear from the proof. Moreover, all the computations in this proof involve a finite number of modes. In this way, this applies for any regularity framework $\mathcal{H}^{s}(0,1)$ where the control system is well-posed.

\subsection{Approximate controllability}

In spite of the lack of exact controllability from the boundary, we now prove that system (12) is approximately controllable. This property is equivalent to a unique continuation property for the adjoint system.

Theorem 9 System 12 is approximately controllable in $L^{2}(0,1)$ for any time $T>0$.

Proof. Thanks to the linearity of system $(12)$, we only have to prove the approximate controllability from the initial state $\left(y^{0}=0, y^{1}=0\right)$. Let us define the map

$$
\Lambda: h \in H^{2}(0, T) \longmapsto\left(y(T), y_{t}(T)\right) \in L^{2}(0,1)^{2} .
$$

We have to prove that the range of this linear operator $\Lambda$ is dense in $L^{2}(0,1)^{2}$. Let $\left(w_{T}^{0}, w_{T}^{1}\right) \in L^{2}(0,1)^{2}$ such that

$$
-\int_{0}^{1} y_{t}(T) w_{T}^{0} d x+\int_{0}^{1} y(T) w_{T}^{1} d x=0 .
$$


Let us define $z_{T}^{0}, z_{T}^{1} \in H^{2} \cap H_{0}^{1}(0,1)$ such that

$$
z_{T}^{0}-\partial_{x x} z_{T}^{0}=w_{T}^{0}, \quad \text { and } \quad z_{T}^{1}-\partial_{x x} z_{T}^{1}=w_{T}^{1},
$$

and consider $z$ as the solution of (13) with initial condition (at $t=T$ ) given by $z_{T}^{0}, z_{T}^{1}$.

We multiply $(12)$ by $z$ and integrate in space and time over $(0,1) \times(0, T)$. Thus, we obtain

$$
\int_{0}^{T}(h(t)+\ddot{h}(t)) z_{x}(1, t) d t=0
$$

where we have used (16) and the fact that $\left(y^{0}=y^{1}=0\right)$. We prove now that we must have $z=0$. This would imply that $z_{T}^{0}=z_{T}^{1}=0$ and consequently $w_{T}^{0}=w_{T}^{1}=0$, which ends the proof. Indeed, let us choose $h(t)=e^{i \frac{2 \pi n}{T} t}$, for $n \in \mathbb{Z}$. Then,

$$
0=\int_{0}^{T}(h(t)+\ddot{h}(t)) z_{x}(1, t) d t=\left(1-\left(\frac{2 \pi n}{T}\right)^{2}\right) \int_{0}^{T} e^{i \frac{2 \pi n}{T} t} z_{x}(1, t) d t
$$

and consequently, the integral term in the right hand side must be zero for any $n \neq \pm \frac{T}{2 \pi}$. Thus, we see that:

- If $\frac{T}{2 \pi} \notin \mathbb{Z}$, then $z_{x}(1, \cdot)$ is orthogonal to any function $e^{i \frac{2 \pi n}{T} t}$. In conclusion, we get $z_{x}(1, \cdot)=0$.

- If $\frac{T}{2 \pi} \in \mathbb{Z}$, then $z_{x}(1, \cdot)$ is orthogonal to any function $e^{i \frac{2 \pi n}{T} t}$ except at most when $n= \pm \frac{T}{2 \pi}$. In conclusion, we get $z_{x}(1, \cdot) \in \operatorname{Span}\left\{e^{i t}, e^{-i t}\right\}$.

In both cases, we can write that there exist $\alpha$ and $\beta \in \mathbb{C}$ such that we have the expression $z_{x}(1, t)=\alpha e^{i t}+\beta e^{-i t}$. In this way, we get

$$
z_{x}(1, t)=\sum_{n \geq 1}\left[\tilde{c}_{n} e^{i \sqrt{\lambda_{n}}(T-t)}+\tilde{d}_{n} e^{-i \sqrt{\lambda_{n}}(T-t)}\right] \sqrt{2}(n \pi)(-1)^{n}=\alpha e^{i t}+\beta e^{-i t},
$$

where we have used (14) with the corresponding coefficients. From this, we see that

$$
\sum_{n \geq 1}\left[\tilde{c}_{n} e^{i \sqrt{\lambda_{n}}(T-t)}+\tilde{d}_{n} e^{-i \sqrt{\lambda_{n}}(T-t)}\right] \sqrt{2}(n \pi)(-1)^{n}-\alpha e^{i t}-\beta e^{-i t}=0,
$$

for all $t \in(0, T)$. As this function is analytic, it vanishes for any $t \in \mathbb{R}$.

By using (17), and noting that $\sqrt{\lambda_{m}} \neq 1$ for any $m \geq 1$, we obtain the following

$$
\begin{array}{r}
0=\lim _{S \rightarrow+\infty} \frac{1}{2 S} \int_{-S}^{S}\left(\sum_{n \geq 1}\left[\tilde{c}_{n} e^{i \sqrt{\lambda_{n}}(T-t)}+\tilde{d}_{n} e^{-i \sqrt{\lambda_{n}}(T-t)}\right] \sqrt{2}(n \pi)(-1)^{n}\right. \\
\left.-\alpha e^{i t}-\beta e^{-i t}\right) e^{i \sqrt{\lambda_{m}} t} d t=(-1)^{m} \sqrt{2}(m \pi) \tilde{c}_{m} e^{i \sqrt{\lambda_{m}} T},
\end{array}
$$

and

$$
\begin{aligned}
0=\lim _{S \rightarrow+\infty} \frac{1}{2 S} \int_{-S}^{S}\left(\sum_{n \geq 1}\left[\tilde{c}_{n} e^{i \sqrt{\lambda_{n}}(T-t)}+\tilde{d}_{n} e^{-i \sqrt{\lambda_{n}}(T-t)}\right]\right. & \sqrt{2}(n \pi)(-1)^{n} \\
\left.-\alpha e^{i t}-\beta e^{-i t}\right) e^{-i \sqrt{\lambda_{m}} t} d t & =(-1)^{m} \sqrt{2}(m \pi) \tilde{d}_{m} e^{-i \sqrt{\lambda_{m}} T} .
\end{aligned}
$$

In consequence, for any $m \geq 1$, we obtain that $\tilde{c}_{m}=\tilde{d}_{m}=0$. This fact implies that $z=0$, which ends the proof of Theorem 9 . 


\section{Moving distributed control on a periodic domain}

The previous results of non exact controllability on a bounded domain can be used to show that the exact controllability of the linearized improved Boussinesq equation with a distributed control supported in a fixed subdomain $\omega \neq \mathbb{T}=\mathbb{R} /(2 \pi \mathbb{Z})$ fails. This lead us to study another type of control whose support moves on the torus $\mathbb{T}=\mathbb{R} /(2 \pi \mathbb{Z})$. This moving control is supposed to be supported in a set that moves at a constant velocity $c$. In this section, we look at the distributed control problem of the improved Boussinesq equation posed on the torus $\mathbb{T}$. Namely, given a time $T>0$, an initial condition $\left(y^{0}, y^{1}\right)$ and a target $\left(y_{T}^{0}, y_{T}^{1}\right)$ on appropriate spaces, we wonder whether we can find a control function $h=h(x, t)$ such that the solution of the following problem

$$
\begin{cases}y_{t t}-y_{x x}-y_{x x t t}=\left(y^{2}\right)_{x x}+b(x+c t) h(x, t), & x \in \mathbb{T}, t>0, \\ y(x, 0)=y^{0}(x), y_{t}(x, 0)=y^{1}(x), & x \in \mathbb{T}\end{cases}
$$

satisfies $y(T)=y_{T}^{0}$ and $y_{t}(T)=y_{T}^{1}$, where $b=b(x)$ is a given nonzero smooth function. In order to deal with the control problem, we first linearize it around the origin to obtain

$$
\begin{cases}y_{t t}-y_{x x}-y_{x x t t}=b(x+c t) h(x, t), & x \in \mathbb{T}, t>0 \\ y(x, 0)=y^{0}(x), y_{t}(x, 0)=y^{1}(x), & x \in \mathbb{T}\end{cases}
$$

We study this linear control system and then we come back to the nonlinear one by means of a fixed-point argument.

\subsection{Well-posedness}

We first study the well-posedness of equation (19) without control,

$$
\begin{cases}y_{t t}-y_{x x t t}-y_{x x}=0, & x \in \mathbb{T}, t>0 \\ y(x, 0)=y^{0}(x), y_{t}(x, 0)=y^{1}(x), & x \in \mathbb{T} .\end{cases}
$$

For $s \geq 0, H^{s}(\mathbb{T})$ is the usual Sobolev space on the torus $\mathbb{T}$, namely

$$
H^{s}(\mathbb{T})=\left\{u: \mathbb{T} \rightarrow \mathbb{R} /\|u\|_{H^{s}(\mathbb{T})}:=\left\|\left(1-\partial_{x}^{2}\right)^{\frac{s}{2}} u\right\|_{L^{2}(\mathbb{T})}<\infty\right\} .
$$

As in the previous section, we easily obtain the following propositions.

Proposition 10 Let $s \geq 0$. For every $\left(y^{0}, y^{1}\right) \in H^{s}(\mathbb{T})^{2}$ the solution $y$ of equation (20) belongs to $C^{1}\left(\left[0,+\infty\left[, H^{s}(\mathbb{T})\right)\right.\right.$ and can be decomposed as

$$
y(x, t)=\left(\alpha_{0}+\beta_{0} t\right)+\sum_{k \in \mathbb{Z}^{*}}\left(\alpha_{k} \cos \left(\sqrt{\frac{k^{2}}{k^{2}+1}} t\right)+\beta_{k} \sqrt{\frac{k^{2}+1}{k^{2}}} \sin \left(\sqrt{\frac{k^{2}}{k^{2}+1}} t\right)\right) e^{i k x} .
$$

where $y^{0}=\sum_{k \in \mathbb{Z}} \alpha_{k} e^{i k x}$ and $y^{1}=\sum_{k \in \mathbb{Z}} \beta_{k} e^{i k x}$. Then for any $T>0$, there exists $C_{0}>0$ such that

$$
\|y\|_{C^{1}\left([0, T], H^{s}(\mathbb{T})\right)} \leq C_{0}\left(\left\|y^{0}\right\|_{H^{s}(\mathbb{T})}+\left\|y^{1}\right\|_{H^{s}(\mathbb{T})}\right) .
$$

Proposition 11 Let $T>0,\left(y^{0}, y^{1}\right) \in H^{s}(\mathbb{T})^{2}$ with $s \geq 0$ and $F \in L^{1}\left(0, T ; H^{s-2}(\mathbb{T})\right)$. Then the solution of the linear system

$$
y_{t t}-y_{x x t t}-y_{x x}=F,
$$

with $y(., 0)=y^{0}$ and $y_{t}(., 0)=y^{1}$, satisfies $y \in C^{1}\left([0, T], H^{s}(\mathbb{T})\right)$ and the solution depends continuously on data, i.e. there exists $C_{2}>0$ such that

$$
\|y\|_{C^{1}\left([0, T], H^{s}(\mathbb{T})\right)} \leq C_{2}\left(\left\|y^{0}\right\|_{H^{s}(\mathbb{T})}+\left\|y^{1}\right\|_{H^{s}(\mathbb{T})}+\|F\|_{L^{1}\left(0, T ; H^{s-2}(\mathbb{T})\right.}\right) .
$$




\subsection{Gap condition}

Let us prove that if we choose $c \in \mathbb{R}$ sufficiently large, the terms $k c \pm \sqrt{\frac{k^{2}}{k^{2}+1}}$ are all different and have an asymptotical gap. This will be useful to study the exact controllability wanted. We define two families of sequences, for $k \in \mathbb{Z}$,

$$
\lambda_{k}^{+}=\left(c k+\frac{|k|}{\sqrt{1+k^{2}}}\right) \text { and } \lambda_{k}^{-}=\left(c k-\frac{|k|}{\sqrt{1+k^{2}}}\right) .
$$

We can easily prove the following lemma concerning the asymptotical gap in these eigenvalues distribution.

Lemma 12 Let us denote by $\Delta$ the asymptotical gap between the eigenvalues. If $\frac{2}{c} \notin \mathbb{Z}$, then

$$
\Delta=|c| \cdot \operatorname{dist}\left(\frac{2}{c}, \mathbb{Z}\right)
$$

Proof. Let $k, k^{\prime} \in \mathbb{N}$. We easily get that

$$
\begin{array}{r}
\lambda_{k^{\prime}}^{+}-\lambda_{k}^{+}=\lambda_{-k}^{-}-\lambda_{-k^{\prime}}^{-}=c\left(k^{\prime}-k\right)-\frac{1}{2 k^{\prime 2}}+\frac{1}{2 k^{2}}+o\left(\frac{1}{k^{2}}\right)+o\left(\frac{1}{k^{\prime 2}}\right) \\
\lambda_{k^{\prime}}^{-}-\lambda_{k}^{+}=c\left(k^{\prime}-k-\frac{2}{c}\right)+\frac{1}{2 k^{\prime 2}}+\frac{1}{2 k^{2}}+o\left(\frac{1}{k^{2}}\right)+o\left(\frac{1}{k^{\prime 2}}\right)
\end{array}
$$

Thus the asymptotical gap is $|c| \cdot \operatorname{dist}\left(\frac{2}{c}, \mathbb{Z}\right)$.

Remark 13 For instance, if $c \geq 4$, then we have an asymptotical gap $\Delta=2$.

\subsection{Exact controllability of the linear system}

We look at the following internal control problem,

$$
\begin{cases}y_{t t}-y_{x x t t}-y_{x x}=b(x+c t) h(x, t), & x \in \mathbb{T}, t>0 \\ y(x, 0)=y^{0}(x), y_{t}(x, 0)=y^{1}(x), & x \in \mathbb{T}\end{cases}
$$

We want to prove the following result, where $\Delta>0$ is defined in Lemma 12 .

Theorem 14 Let $s \geq 2$ and $c$ be such that $|c|>2$. Let $b=b(x) \in C^{\infty}(\mathbb{T})$ be such that $\{x \in \mathbb{T}, b(x) \neq 0\} \neq \emptyset$. Then, for all $T>\frac{2 \pi}{\Delta}$ and all $\left(y^{0}, y^{1}\right),\left(y_{T}^{0}, y_{T}^{1}\right) \in H^{s}(\mathbb{T}) \times H^{s}(\mathbb{T})$, there exists a control $h \in L^{2}\left(0, T ; H^{s-2}(\mathbb{T})\right)$ such that the linear problem (21) admits a unique solution $y \in C^{1}\left([0, T], H^{s}(\mathbb{T})\right)$ such that $y(x, T)=y_{T}^{0}(x)$ and $y_{t}(x, T)=y_{T}^{1}(x)$. Furthermore, there exists $C_{1}>0$ such that

$$
\|h\|_{L^{2}\left(0, T, H^{s-2}(\mathbb{T})\right)} \leq C_{1}\left(\left\|\left(y^{0}, y^{1}\right)\right\|_{H^{s}(\mathbb{T})^{2}}+\left\|\left(y_{T}^{0}, y_{T}^{1}\right)\right\|_{H^{s}(\mathbb{T})^{2}}\right)
$$

Remark 15 The condition $|c|>2$ is useful in two ways. To be sure the asymptotic gap $\Delta$ is positive $\left(\frac{2}{c} \notin \mathbb{Z}\right)$ and to avoid the existence of different $k, m$ such that

$$
c k+\sqrt{\frac{k^{2}}{k^{2}+1}}=c m-\sqrt{\frac{m^{2}}{m^{2}+1}} .
$$

The latter is needed in order to solve the moment problem with no additional compatibility conditions on the initial and final data. 
Proof. The adjoint problem is written as follows,

$$
\varphi_{t t}-\varphi_{x x t t}-\varphi_{x x}=0, \quad x \in \mathbb{T}, t>0 .
$$

We easily remark that if $y$ is solution of the direct problem (21) with $h=0$, then $\varphi(x, t)=y(2 \pi-x, T-t)$ is a solution of the adjoint problem $(22)$.

Let us multiply equation (21) by $\bar{\varphi}$ where $\varphi$ is a solution of (22) and integrate by parts on $[0, T] \times \mathbb{T}$. Then, we obtain

$$
\left.\int_{\mathbb{T}}\left[y_{t}\left(\bar{\varphi}-\bar{\varphi}_{x x}\right)-y\left(\bar{\varphi}_{t}-\bar{\varphi}_{x x t}\right)\right]\right|_{t=0} ^{T} d x=\int_{0}^{T} \int_{\mathbb{T}} h(x, t) b(x+c t) \bar{\varphi}(x, t) d x d t .
$$

We take, for $k \in \mathbb{Z}, \bar{\varphi}^{+}(x, t)=e^{i \sqrt{\frac{k^{2}}{k^{2}+1}}(T-t)} e^{-i k x}$ and $\bar{\varphi}^{-}(x, t)=e^{-i \sqrt{\frac{k^{2}}{k^{2}+1}}(T-t)} e^{-i k x}$. Thus, 23) becomes

$$
\begin{array}{r}
\left(1+k^{2}\right)\left(\left\langle y_{t}(T)-e^{ \pm i \sqrt{\frac{k^{2}}{k^{2}+1}} T} y_{t}(0), e^{i k x}\right\rangle \pm i \sqrt{\frac{k^{2}}{k^{2}+1}}\left\langle y(T)-e^{ \pm i \sqrt{\frac{k^{2}}{k^{2}+1}} T} y(0), e^{i k x}\right\rangle\right) \\
=\int_{0}^{T} \int_{\mathbb{T}} h(x, t) b(x+c t) e^{ \pm i \sqrt{\frac{k^{2}}{k^{2}+1}}(T-t)} e^{-i k x} d x d t .
\end{array}
$$

where $\left\langle f, e^{i k x}\right\rangle$ stands for the coordinate $f_{k}$ in the Fourier decomposition $f=\sum_{k \in \mathbb{Z}} f_{k} e^{i k x}$.

Taking $\varphi=t$ in $(23)$, we obtain

$$
T \int_{\mathbb{T}} y_{t}(T) d x-\int_{\mathbb{T}} y(T) d x+\int_{\mathbb{T}} y(0) d x=\int_{0}^{T} \int_{\mathbb{T}} h(x, t) b(x+c t) t d x d t .
$$

By a simple change of variables we can rewrite the right hand side of (24) and (25) as follows,

$$
\begin{gathered}
\int_{0}^{T} \int_{\mathbb{T}} h(x, t) b(x+c t) e^{ \pm i \sqrt{\frac{k^{2}}{k^{2}+1}}(T-t)} e^{-i k x} d x d t \\
=\int_{0}^{T} \int_{\mathbb{T}} h(x-c t, t) b(x) e^{ \pm i \sqrt{\frac{k^{2}}{k^{2}+1}}(T-t)} e^{-i k(x-c t)} d x d t, \\
\int_{0}^{T} \int_{\mathbb{T}} h(x, t) b(x+c t) t d x d t=\int_{0}^{T} \int_{\mathbb{T}} h(x-c t, t) b(x) t d x d t .
\end{gathered}
$$

Let us define $\tilde{h}(x, t)=h(x-c t, t)$. Then the moment problem consists in finding a control $\tilde{h}$ such that for all $k \in \mathbb{Z}$,

$$
\begin{aligned}
\left(1+k^{2}\right)\left(\left\langle e^{\mp i \sqrt{\frac{k^{2}}{k^{2}+1}} T} y_{t}(T)-y_{t}(0), e^{i k x}\right\rangle\right. & \left. \pm i \sqrt{\frac{k^{2}}{k^{2}+1}}\left\langle e^{\mp i \sqrt{\frac{k^{2}}{k^{2}+1}} T} y(T)-y(0), e^{i k x}\right\rangle\right) \\
& =\int_{0}^{T} \int_{\mathbb{T}} \tilde{h}(x, t) b(x) e^{i\left(k c \mp \sqrt{\frac{k^{2}}{k^{2}+1}}\right) t} e^{-i k x} d x d t
\end{aligned}
$$


and

$$
T \int_{\mathbb{T}} y_{t}(T) d x-\int_{\mathbb{T}} y(T) d x+\int_{\mathbb{T}} y(0) d x=\int_{0}^{T} \int_{\mathbb{T}} \tilde{h}(x, t) b(x) t d x d t .
$$

Because of the asymptotical gap $\Delta$ and the fact that the time exponential functions are all different, we can apply standard results on complex exponential functions to prove, for any $T>\frac{2 \pi}{\Delta}$, the existence of a function $h \in L^{2}\left(0, T, H^{s-2}(\mathbb{T})\right)$ solving (26)-(27). Indeed, we choose $\left\{q_{0}, \tilde{q}_{0}\right\} \cup\left\{q_{k}^{ \pm}\right\}_{k \in \mathbb{Z}^{*}} \subset L^{2}(0, T)$, as a biorthogonal family to the set

$$
S=\{1, t\} \cup\left\{e^{i\left(k c \mp \sqrt{\frac{k^{2}}{k^{2}+1}}\right) t}\right\}_{k \in \mathbb{Z}^{*}} .
$$

Remark 16 The existence of this biorthogonal family can be established as explained in [17, section 2]. The set $S$ is not complete in $L^{2}(0, T)$ under the condition

$$
\limsup _{y \rightarrow \infty} \limsup _{x \rightarrow \infty} \frac{\Lambda(x+y)-\Lambda(x)}{y}<\frac{T}{2 \pi}
$$

where $\Lambda(x)$ denotes the number of $\left(k c \mp \sqrt{\frac{k^{2}}{k^{2}+1}}\right)$ which are less than $x$. This condition holds given the structure of $\left\{k c \mp \sqrt{\frac{k^{2}}{k^{2}+1}}\right\}_{k \in \mathbb{Z}^{*}}$ and our choice of $T$. The existence of the biorthogonal family is deduced from the lack of completeness. In particular, to deal with the function $t$, one can use that for $\omega>0$

$$
\lim _{\omega \rightarrow 0^{+}} \frac{e^{i \omega t}-e^{-i \omega t}}{2 i \omega}=t, \quad \text { uniformly in }[0, T] .
$$

We follow [16], and look for a control in the form

$$
\tilde{h}(x, t)=b(x)\left\{f_{0} q_{0}(t)+\tilde{f}_{0} \tilde{q}_{0}(t)+\sum_{j \in \mathbb{Z}^{*}}\left(f_{j}^{+} q_{j}^{+}(t)+f_{j}^{-} q_{j}^{-}(t)\right) e^{i j x}\right\}
$$

where $f_{0}, \tilde{f}_{0}, f_{j}^{ \pm}$are scalars chosen to satisfy the moment problem 26)-27). Thus, we obtain, for $k \in \mathbb{Z}^{*}$,

$$
\begin{aligned}
f_{k}^{+}=\frac{\left(1+k^{2}\right)}{\int_{\mathbb{T}} b^{2}(x) d x}\left(\left\langlee^{-i \sqrt{\frac{k^{2}}{k^{2}+1}} T} y_{t}(T)\right.\right. & \left.-y_{t}(0), e^{i k x}\right\rangle \\
& \left.+i \sqrt{\frac{k^{2}}{k^{2}+1}}\left\langle e^{-i \sqrt{\frac{k^{2}}{k^{2}+1}} T} y(T)-y(0), e^{i k x}\right\rangle\right),
\end{aligned}
$$

$$
\begin{aligned}
f_{k}^{-}=\frac{\left(1+k^{2}\right)}{\int_{\mathbb{T}} b^{2}(x) d x}\left(\left\langle e^{i \sqrt{\frac{k^{2}}{k^{2}+1}} T} y_{t}(T)-y_{t}(0), e^{i k x}\right\rangle\right. \\
\left.-i \sqrt{\frac{k^{2}}{k^{2}+1}}\left\langle e^{i \sqrt{\frac{k^{2}}{k^{2}+1}} T} y(T)-y(0), e^{i k x}\right\rangle\right),
\end{aligned}
$$

and

$$
f_{0}=\frac{1}{\int_{\mathbb{T}} b^{2}(x) d x}\left(\int_{\mathbb{T}} y_{t}(T) d x-\int_{\mathbb{T}} y_{t}(0) d x\right)
$$




$$
\tilde{f}_{0}=\frac{1}{\int_{\mathbb{T}} b^{2}(x) d x}\left(T \int_{\mathbb{T}} y_{t}(T) d x-\int_{\mathbb{T}} y(T) d x+\int_{\mathbb{T}} y(0) d x\right),
$$

where $\int_{\mathbb{T}} b^{2}(x) d x \neq 0$ by hypothesis. Furthermore, from equations $28-(29)-(30)-31$ we obtain the continuity of the control with respect to data. More explicitly, we obtain the existence of a constant $C>0$ such that

$$
\begin{aligned}
\|\tilde{h}\|_{L^{2}\left(0, T ; H^{s-2}(\mathbb{T})\right)}^{2} & =\int_{0}^{T}\left\|b(x)\left\{f_{0} q_{0}(t)+\tilde{f}_{0} \tilde{q}_{0}(t)+\sum_{j \in \mathbb{Z}^{*}}\left(f_{j}^{+} q_{j}^{+}(t)+f_{j}^{-} q_{j}^{-}(t)\right) e^{i j x}\right\}\right\|_{H^{s-2}(\mathbb{T})}^{2} d t \\
& \leq C \int_{0}^{T}\left\{\left|f_{0} q_{0}(t)\right|^{2}+\left|\tilde{f}_{0} \tilde{q}_{0}(t)\right|^{2}+\sum_{j \in \mathbb{Z}^{*}}\left(1+j^{2}\right)^{s-2}\left|f_{j}^{+} q_{j}^{+}(t)+f_{j}^{-} q_{j}^{-}(t)\right|^{2}\right\} d t \\
& \leq 2 C\left\{\left|f_{0}\right|^{2}+\left|\tilde{f}_{0}\right|^{2}+\sum_{j \in \mathbb{Z}^{*}}\left(1+j^{2}\right)^{s-2}\left(\left|f_{j}^{+}\right|^{2}+\left|f_{j}^{-}\right|^{2}\right)\right\} \\
& \leq 2 C\left(\left\|y^{0}\right\|_{H^{s}(\mathbb{T})}^{2}+\left\|y^{1}\right\|_{H^{s}(\mathbb{T})}^{2}+\left\|y_{T}^{0}\right\|_{H^{s}(\mathbb{T})}^{2}+\left\|y_{T}^{1}\right\|_{H^{s}(\mathbb{T})}^{2}\right)
\end{aligned}
$$

Then, $h(x, t)=\tilde{h}(x+c t, t)$ is the desired control function that drives the system from $\left(y^{0}, y^{1}\right)$ to $\left(y_{T}^{0}, y_{T}^{1}\right)$, which concludes the proof.

\subsection{Local exact controllability of the nonlinear system}

We follow the proof of the local exact controllability of the Boussinesq equation given in [6]. We decompose the solution of the nonlinear problem,

$$
\begin{cases}y_{t t}-y_{x x}-y_{x x t t}=\left(y^{2}\right)_{x x}+b(x+c t) h(x, t), & x \in \mathbb{T}, t>0 \\ y(x, 0)=y^{0}(x), y_{t}(x, 0)=y^{1}(x), & x \in \mathbb{T}\end{cases}
$$

in the following way, $y=\alpha+\beta+\gamma$, where $\alpha$ is the solution of the linear problem with initial data and with no control,

$$
\begin{cases}\alpha_{t t}-\alpha_{x x}-\alpha_{x x t t}=0, & x \in \mathbb{T}, t>0 \\ \alpha(x, 0)=y^{0}(x), \alpha_{t}(x, 0)=y^{1}(x), & x \in \mathbb{T}\end{cases}
$$

$\beta$ is the solution of the linear problem with control but with null initial data,

$$
\begin{cases}\beta_{t t}-\beta_{x x}-\beta_{x x t t}=b(x+c t) h(x, t), & x \in \mathbb{T}, t>0 \\ \beta(x, 0)=0, \beta_{t}(x, 0)=0, & x \in \mathbb{T}\end{cases}
$$

and $\gamma$ is the solution of the linear problem with a second member term $F$,

$$
\begin{cases}\gamma_{t t}-\gamma_{x x}-\gamma_{x x t t}=F, & x \in \mathbb{T}, t>0 \\ \gamma(x, 0)=0, \gamma_{t}(x, 0)=0, & x \in \mathbb{T}\end{cases}
$$

(Later, the source term $F$ will be taken as the nonlinearity $\left(y^{2}\right)_{x x}$.)

We study the nonlinear problem in the following regularity framework:

$$
\left(y^{0}, y^{1}\right) \in H^{2}(\mathbb{T})^{2}, h \in L^{2}\left(0, T, L^{2}(\mathbb{T})\right), F \in L^{1}\left(0, T ; L^{2}(\mathbb{T})\right) \text { and } b \in C^{\infty}(\mathbb{T}),
$$

where $b$ is such that $\{x \in \mathbb{T}, b(x) \neq 0\} \neq \emptyset$.

Let us consider the following maps, which are well-defined, linear and continuous by Propositions 10 and 11 . 
- The initial data-to-solution map:

$$
\psi_{0}:\left(y^{0}, y^{1}\right) \in H^{2}(\mathbb{T})^{2} \longmapsto \alpha \in C^{1}\left([0, T], H^{2}(\mathbb{T})\right),
$$

where $\alpha$ is the solution of (34).

- The control-to-solution map:

$$
\psi_{1}: h \in L^{2}\left(0, T, L^{2}(\mathbb{T})\right) \longmapsto \beta \in C^{1}\left([0, T], H^{2}(\mathbb{T})\right),
$$

where $\beta$ is the solution of 35 .

- The source term-to-solution map:

$$
\psi_{2}: F \in L^{1}\left(0, T ; L^{2}(\mathbb{T})\right) \longmapsto \gamma \in C^{1}\left([0, T], H^{2}(\mathbb{T})\right),
$$

where $\gamma$ is the solution of $(36)$.

In order to deal with the nonlinearity, we need the following proposition.

Proposition 17 ([6]) The map

$$
\phi \in L^{2}\left(0, T, H^{2}(\mathbb{T})\right) \longmapsto\left(\phi^{2}\right)_{x x} \in L^{1}\left(0, T, L^{2}(\mathbb{T})\right),
$$

is well-defined and continuous. We have the existence of $K>0$ such that

$\left\|\left(\phi^{2}\right)_{x x}-\left(\psi^{2}\right)_{x x}\right\|_{L^{1}\left(0, T, L^{2}(\mathbb{T})\right)} \leq K\left(\|\phi\|_{L^{2}\left(0, T, H^{2}(\mathbb{T})\right)}+\|\psi\|_{L^{2}\left(0, T, H^{2}(\mathbb{T})\right)}\right)\|\phi-\psi\|_{L^{2}\left(0, T, H^{2}(\mathbb{T})\right)}$.

Proof. This proof is exactly the same as in [6, Proposition 6], where the result is obtained in the spatial domain $[0, L]$.

Thanks to equation 32 , we can define a continuous map

$$
\Gamma:\left(y_{T}^{0}, y_{T}^{1}\right) \in H^{2}(\mathbb{T})^{2} \longmapsto h \in L^{2}\left(0, T, L^{2}(\mathbb{T})\right),
$$

such that the solution $\beta \in C^{1}\left([0, T], H^{2}(\mathbb{T})\right)$ of 35 satisfies $\left(\beta(T), \beta_{t}(T)\right)=\left(y_{T}^{0}, y_{T}^{1}\right)$. Let us define the following map

$$
\Pi: y \in C^{1}\left([0, T], H^{2}(\mathbb{T})\right) \mapsto \Pi(y) \in C^{1}\left([0, T], H^{2}(\mathbb{T})\right)
$$

where

$$
\begin{aligned}
& \Pi(y)=\psi_{0}\left(y^{0}, y^{1}\right)+\psi_{2}\left(\left(y^{2}\right)_{x x}\right) \\
+ & \psi_{1} \circ \Gamma\left(\left(y_{T}^{0}, y_{T}^{1}\right)-\left(\psi_{0}\left(y^{0}, y^{1}\right)(T), \psi_{0 t}\left(y^{0}, y^{1}\right)(T)\right)-\left(\psi_{2}\left(\left(y^{2}\right)_{x x}\right)(T), \psi_{2 t}\left(\left(y^{2}\right)_{x x}\right)(T)\right)\right) .
\end{aligned}
$$

We see that a fixed-point of $\Pi$ is a trajectory $y=y(x, t)$ of $(33)$ going from $\left(y^{0}, y^{1}\right)$ at time $t=0$ to $\left(y_{T}^{0}, y_{T}^{1}\right)$ at time $t=T$.

Let us apply the Banach fixed-point theorem. We consider small data with $\epsilon>0$ to be fixed later:

$$
\left\|\left(y^{0}, y^{1}\right)\right\|_{H^{2}(\mathbb{T})^{2}} \leq \epsilon, \quad\left\|\left(y_{T}^{0}, y_{T}^{1}\right)\right\|_{H^{2}(\mathbb{T})^{2}} \leq \epsilon .
$$

We have to find $R>0$ and $D \in(0,1)$ such that 
1. $\Pi\left(B_{R}\right) \subset B_{R}$ where $B_{R}$ is the closed ball

$$
B_{R}=\left\{v \in C^{1}\left([0, T], H^{2}(\mathbb{T})\right) /\|v\| \leq R\right\} .
$$

(In this section, $\|\cdot\|$ stands for the norm in $C^{1}\left([0, T], H^{2}(\mathbb{T})\right)$.)

2. $\|\Pi(v)-\Pi(w)\| \leq D\|v-w\|$.

Taking in mind the constants $C_{0}, C_{2}, K, C_{1}$ in Propositions 10, 11, 17 and Theorem 14 . respectively, we obtain for $v \in B_{R}$

$$
\|\Pi(v)\| \leq C_{0} \epsilon+C_{2} C_{1}\left(\epsilon+C_{0} \epsilon+C_{2} K R^{2}\right)+C_{2} K R^{2} .
$$

Thus, we obtain the first condition to satisfy:

$$
C_{0} \epsilon+C_{2} C_{1}\left(\epsilon+C_{0} \epsilon+C_{2} K R^{2}\right)+C_{2} K R^{2} \leq R .
$$

On the other hand, for $v, w \in B_{R}$,

$\Pi(v)-\Pi(w)=\psi_{1} \circ \Gamma\left(\psi_{2}\left(\left(w^{2}\right)_{x x}-\left(v^{2}\right)_{x x}\right)(T), \psi_{2 t}\left(\left(w^{2}\right)_{x x}-\left(v^{2}\right)_{x x}\right)(T)\right)-\psi_{2}\left(\left(w^{2}\right)_{x x}-\left(v^{2}\right)_{x x}\right)$, and then

$$
\|\Pi(v)-\Pi(w)\| \leq 2 C_{2} C_{1} C_{2} K R\|v-w\|+2 C_{2} K R\|v-w\| \leq 2 \tilde{C} R\|v-w\| .
$$

In this way, we obtain the second condition to satisfy:

$$
\tilde{C} R<1 / 2 .
$$

By chosing $\epsilon, R>0$ small enough in order to satisfy (37) and (38), we can apply the Banach fixed-point theorem to prove the following result.

Theorem 18 Let $c$ be such that $|c|>2$. Let $b=b(x) \in C^{\infty}(\mathbb{T})$ be such that $\{x \in$ $\mathbb{T}, b(x) \neq 0\} \neq \emptyset$. Then, for all $T>\frac{2 \pi}{\Delta}$, there exists $\epsilon>0$ such that for any $\left(y^{0}, y^{1}\right),\left(y_{T}^{0}, y_{T}^{1}\right) \in H^{2}(\mathbb{T})^{2}$ satisfying

$$
\left\|\left(y^{0}, y^{1}\right)\right\|_{H^{2}(\mathbb{T})^{2}} \leq \epsilon, \quad\left\|\left(y_{T}^{0}, y_{T}^{1}\right)\right\|_{H^{2}(\mathbb{T})^{2}} \leq \epsilon,
$$

there exists a control $h \in L^{2}\left(0, T, L^{2}(\mathbb{T})\right)$ such that the problem (33) admits a unique solution $y \in C^{1}\left([0, T], H^{2}(\mathbb{T})\right)$ such that

$$
y(x, T)=y_{T}^{0}(x), \text { and } y_{t}(x, T)=y_{T}^{1}(x) .
$$

\section{Conclusion}

In this paper we have studied several controllability properties for the improved Boussinesq equation, posed either on a bounded interval or on the torus. If the control acts on the boundary or is distributed on a fixed subdomain, some non exact controllability results appear for the linearized equation due to the existence of an accumulation point in the spectrum of the underlying operator. In order to obtain exact controllability results for both linear and nonlinear equations, we consider the case of a moving control. Here, we require the speed at which the control domain moves to be large enough. 
For this equation, a natural open problem appears: can we stabilize the system? On a bounded domain with either homogeneous Dirichlet boundary conditions or periodic boundary conditions, the solution of

$$
y_{t t}-y_{x x t t}-y_{x x}=0
$$

conserves the following energy

$$
E(t)=\frac{1}{2} \int\left\{\left|y_{t}(t, x)\right|^{2}+\left|y_{t x}(t, x)\right|^{2}+\left|y_{x}(t, x)\right|^{2}\right\} d x .
$$

Thus, we wonder what kind of internal or boundary damping mechanisms stabilizes the system. In the internal control case, some terms as $y_{t}$ and $-y_{x x t}$ should help to get a decreasing energy. See [19] where the same kind of problems are studied for the stabilization of the Benjamin-Bona-Mahony equation.

\section{References}

[1] J. Boussinesq, Théorie des ondes et des remous qui se propagent le long d'un canal rectangulaire horizontal, en communiquant au liquide contenu dans ce canal des vitesses sensiblement pareilles de la surface au fond, J. Math. Pures Appl. Ser., 2 (1872), pp. 55-108.

[2] C. Castro, Exact controllability of the 1-D wave equation from a moving interior point, ESAIM Control Optim. Calc. Var., 19 (2013), pp. 301-316.

[3] C. Castro, N. Cîndea, And A. Münch, Controllability of the linear onedimensional wave equation with inner moving forces, SIAM J. Control Optim., 52 (2014), pp. 4027-4056.

[4] C. Castro And E. Zuazua, Unique continuation and control for the heat equation from an oscillating lower dimensional manifold, SIAM J. Control Optim., 43 (2004/05), pp. 1400-1434 (electronic).

[5] F. W. Chaves-Silva, L. Rosier, and E. Zuazua, Null controllability of a system of viscoelasticity with a moving control, J. Math. Pures Appl. (9), 101 (2014), pp. 198-222.

[6] E. CRÉPeAu, Exact controllability of the Boussinesq equation on a bounded domain, Differential Integral Equations, 16 (2003), pp. 303-326.

[7] A. Khapalov, Mobile point controls versus locally distributed ones for the controllability of the semilinear parabolic equation, SIAM J. Control Optim., 40 (2001), pp. 231-252 (electronic).

[8] A. Y. Khapalov, Controllability of the wave equation with moving point control, Appl. Math. Optim., 31 (1995), pp. 155-175.

[9] V. Komornik and P. Loreti, Fourier series in control theory, Springer Monographs in Mathematics, Springer-Verlag, New York, 2005.

[10] G. Leugering And E. J. P. G. Schmidt, Boundary control of a vibrating plate with internal damping, Math. Methods Appl. Sci., 11 (1989), pp. 573-586. 
[11] J.-L. Lions, Pointwise control for distributed systems, in: H.T. Banks (Ed.), Control and Estimation in Distributed Parameter Systems, SIAM (1992).

[12] V. Makhankov, Dynamics of classical solitons (in non-integrable systems), Physics reports, 35 (1978), pp. 1-128.

[13] P. Martin, L. Rosier, And P. Rouchon, Null controllability of the structurally damped wave equation with moving control, SIAM J. Control Optim., 51 (2013), pp. 660-684.

[14] S. Micu, On the controllability of the linearized Benjamin-Bona-Mahony equation, SIAM J. Control Optim., 39 (2001), pp. 1677-1696 (electronic).

[15] L. Rosier And P. Rouchon, On the controllability of a wave equation with structural damping, Int. J. Tomogr. Stat, 5 (2007), pp. 79-84.

[16] L. Rosier AND B.-Y. Zhang, Unique continuation property and control for the Benjamin-Bona-Mahony equation on a periodic domain, J. Differential Equations, 254 (2013), pp. 141-178.

[17] D. L. Russell, Nonharmonic Fourier series in the control theory of distributed parameter sytems, J. Math. Anal. Appl., 18 (1967), pp. 542-560.

[18] —, Mathematical models for the elastic beam and their control-theoretic implications, in Semigroups, theory and applications, Vol. II (Trieste, 1984), vol. 152 of Pitman Res. Notes Math. Ser., Longman Sci. Tech., Harlow, 1986, pp. 177-216.

[19] X. Zhang And E. Zuazua, Unique continuation for the linearized benjamin-bonamahony equation with space-dependent potential, Math. Ann., 325 (2003), pp. 543582 .

[20] Y. ZHIJIAN, Existence and non-existence of global solutions to a generalized modification of the improved Boussinesq equation, Math. Methods Appl. Sci., 21 (1998), pp. 1467-1477. 